\title{
Tumor specific liposomes improve detection of pancreatic adenocarcinoma in vivo using optoacoustic tomography
}

\author{
Wenyuan Yin ${ }^{1}$, Charles W. Kimbrough', Jorge G. Gomez-Gutierrez ', Christopher T. Burns ${ }^{1}$, Phillip Chuong ${ }^{1}$, \\ William E. Grizzle ${ }^{2}$ and Lacey R. McNally ${ }^{1 *}$
}

\begin{abstract}
Background: Pancreatic cancer often goes undiagnosed until late stage disease due in part to suboptimal early detection. Our goal was to develop a Syndecan-1 tagged liposome containing fluorescent dye as an improved contrast agent for detection of pancreatic adenocarcinoma in vivo using multispectral optoacoustic tomography.

Results: The diagnostic capabilities and specificity to pancreatic adenocarcinoma of Syndecan-1 targeted liposomes were evaluated both in vitro and in vivo. Immunocytochemistry showed that liposomes preferentially bound to and released their contents into cells expressing high levels of insulin-like growth factor 1 receptor. We determined that the contents of the liposome were released into the cell as noted by the change in propidium iodide fluorescence from green to red based upon nucleic acid binding. In an orthotopic mouse model, the liposomes preferentially targeted the pancreatic tumor with little off-target binding in the liver and spleen. Peak accumulation of the liposomes in the tumor occurred at $8 \mathrm{~h}$ post-injection. Multispectral optoacoustic tomographic imaging was able to provide high-resolution 3D images of the tumor and liposome location. Ex vivo analysis showed that non-targeted liposomes accumulated in the liver, suggesting that specificity of the liposomes for pancreatic adenocarcinoma was due to the presence of the Syndecan-1 ligand.

Conclusions: This study demonstrated that Syndecan-1 liposomes were able to release cargo into IGF1-R expressing tumor cells. The Syndecan-1 liposomes demonstrated tumor specificity in orthotopic pancreatic cancer as observed using multispectral optoacoustic tomography with reduced kidney and liver uptake. By targeting the liposome with Syndecan-1, this nanovehicle has potential as a targeted theranostic nanoparticle for both drug and contrast agent delivery to pancreatic tumors.
\end{abstract}

\section{Background}

Pancreatic adenocarcinoma has retained a high mortality rate due to inadequate early detection methods and the relative ineffectiveness of current therapy regimens [1-4]. In particular, the underlying biology of pancreatic ductal adenocarcinoma can make these tumors particularly resistant to chemotherapy. The dense desmoplastic stroma and poor vascularity of these tumors inhibits the accumulation of traditional chemotherapies, and prevents therapeutic drug concentrations from being

\footnotetext{
*Correspondence: Lrmcna01@louisville.edu

1 University of Louisville, 505 S Hancock, Louisville, KY 40202, USA Full list of author information is available at the end of the article
}

realized within the tumor [5]. Nanoparticle drug carriers are an effective strategy to overcome these extracellular barriers to drug delivery, and can increase the therapeutic load at tumor sites while decreasing systemic toxicity [6-8]. Liposomes in particular have multiple desirable characteristics, such as the capacity for encapsulating large amounts of materials, the ability to protect these materials from degradation, and the capability for intracellular delivery through fusion with the plasma membrane [9].

In addition to being drug carriers, liposomes can also encapsulate contrast agents for diagnostic imaging, combining diagnostic and therapeutic functions into one system. These particles, termed "theranostic nanoparticles", 
are a novel approach for detecting, monitoring, and treating cancer $[10,11]$. The contrast agents loaded into theranostic nanoparticles allow nanoparticle accumulation and biodistribution to be tracked using standard techniques such as planar fluorescent imaging [12].

However, multi-spectral optoacoustic tomography (MSOT) offers several advantages over traditional imaging modalities. MSOT operates via the photoacoustic effect, wherein a medium excited by pulsed laser light subsequently emits an acoustic wave. Optically active molecules, or chromophores, absorb a photon and enter an excited state, generating heat upon relaxation. This pulse of heat leads to an increase in temperature and local pressure, termed thermoelastic expansion. Propagation of the pressure differential results in the formation of an acoustic wave, which can then be recorded with ultrasound detectors to create high-resolution 3D images [13, 14]. Expansion of endogenous and exogenous chromophores occur at specific excitation wavelengths, allowing for selective imaging of compounds of interest. Due to the low-scattering characteristic of ultrasonic waves, images do not suffer from the light scattering or signal attenuation that limits optical and fluorescent imaging. Additional advantages include an enhanced spatial resolution of up to $150 \mu \mathrm{m}$ and accurate 3D imaging of deep tissue layers [14-18]. Furthermore, multi-wavelength measurements can be used to quantify spatially varying concentrations of chromophores within biological tissues [13]. MSOT can therefore be a valuable tool in determining the precise location and relative concentration of theranostic nanoparticles in vivo.

Syndecan-1 (Sdc1) has been shown to bind specifically to pancreatic adenocarcinoma, making it a promising targeting ligand for a theranostic nanoparticle $[19,20]$. This study examines the feasibility of using Syndecan-1 tagged liposomes as a theranostic nanoparticle for pancreatic adenocarcinoma, using MSOT to evaluate the specificity of these targeted liposomes for pancreatic adenocarcinoma in vivo.

\section{Methods}

\section{Cell culture}

Pancreatic adenocarcinoma cell lines S2VP10 and S2013, metastatic subclones of the SUIT-2 line, were obtained from Dr. Michael A. Hollingsworth at the University of Nebraska. Squamous cell carcinoma cell line SCC-1 was obtained from the University of Michigan. Pancreatic adenocarcinoma cell line MiaPaCa-2 was obtained from ATCC (Manassas, VA, USA). S2VP10, S2013, and SCC-1 cells were cultured in RPMI 1640 and MiaPaCa-2 cells in DMEM medium (Gibco, Grand Island, NY, USA), supplemented with $10 \%$ Fetal Bovine Serum (Atlanta Biologicals, Flowery Branch, GA, USA) and $1 \%$ L-Glutamine
(Gibco). The cells were cultured at $37{ }^{\circ} \mathrm{C}$ and $5 \% \mathrm{CO}_{2}$. S2VP10 cells were infected with a retrovirus containing the firefly luciferase (Luc) gene (Stratagene, La Jolla, CA, USA) and a luciferase-positive single cell clone, S2VP10L, was isolated [21].

\section{Synthesis of liposomes \\ Materials}

Soybean L- $\alpha$-phosphatidylcholine (95\%) (PC), 1,2-dioleoylsn-glycero-3-phosphoethanolamine (DOPE) and 1,2-dioleoyl-sn-glycero-3-phosphoethanolamine- $N$-(hexanoylamine) (CAP) were purchased from Avanti Polar Lipids (Alabaster, AL, USA) (Additional file 1: Figure S1). N-Hydroxysulfosuccinimide sodium salt (NHS), $N$-(3-dimethylaminopropyl)$N^{\prime}$-ethylcarbodiimide hydrochloride (EDC) and propidium iodide were purchased from Sigma (St. Louis, MO, USA). CF-750 N-hydroxysuccinimide ester amine-reactive dye was purchased from Biotium (Hayward, CA, USA). Finally, Syndecan-1 (Sdc1) was purchased from Prospec (Rehovot, Israel).

\section{Synthesis of propidium iodide and CF-750 encapsulated liposomes}

Naked liposomes were synthesized by thin film hydration method [22]. Soybean PC (0.0067 g), CAP (0.0023 g) and DOPE $(0.0020 \mathrm{~g})$ were dissolved in chloroform. Then, the chloroform was evaporated by heating at $70{ }^{\circ} \mathrm{C}$ for $10 \mathrm{~min}$ in a rotary evaporator to get a thin film of lipids. The thin film was hydrated by adding $1 \mathrm{~mL}$ PBS ( $\mathrm{pH} 7.4,0.9 \%$ $\mathrm{NaCl})$ and propidium iodide or CF-750 (1 mM, $0.1 \mathrm{~mL})$ for $2 \mathrm{~h}$ at $70^{\circ} \mathrm{C}$. Then, the liposomes were sonicated for $2 \mathrm{~h}$ at $70^{\circ} \mathrm{C}$. Finally, liposomes were extruded four times through a polycarbonate filter $(200 \mathrm{~nm})$ at $70{ }^{\circ} \mathrm{C}$.

\section{Conjugation of Syndecan-1 to dye-encapsulated liposomes}

Syndecan-1 was conjugated to the dye-encapsulated naked liposomes by carbodiimide chemistry. Syndecan-1 $(0.74 \mathrm{mM}, 20 \mu \mathrm{l})$ was mixed with NHS $(1 \mathrm{mM}, 0.1 \mathrm{~mL})$ and EDC ( $1 \mathrm{mM}, 0.1 \mathrm{~mL})$ and incubated for $6 \mathrm{~h}$ at room temperature at $\mathrm{pH}$ 7.5. Then, $1 \mathrm{~mL}$ of liposomes was added to this solution and mixed for 1 day at room temperature. The excess NHS, EDC, and Sdc1 were removed by dialysis, minimum 2000-3000 Da. The stock solution of Sdc1 conjugated liposomes was diluted further to get the desired concentration.

\section{Characterization of liposomes DLS measurements}

The size distribution of the liposomes was analyzed by dynamic light scattering (DLS) using a Zetasizer NanoZS (Malvern Instruments Ltd., Malvern, UK). The detection angle was 173 degrees and the incident beam was a $\mathrm{He}-\mathrm{Ne}$ ion laser $(\lambda=633 \mathrm{~nm})$. Correlation functions 
were analyzed by a histogram method and used to determine the diffusion coefficient (D) of the micelles in the sample. Hydrodynamic radius (Rh) was calculated from $D$ by using Stokes-Einstein's equation:

$$
R_{h}=\frac{K_{b} T}{6 \pi \eta D}
$$

where $\mathrm{kB}$ is Boltzmann's constant, $\mathrm{T}$ is absolute temperature, and $\eta$ is solvent viscosity [23].

\section{Zeta-potential measurements}

Electrophoretic mobility $(\mu \mathrm{E})$ of the liposomes was measured at $25{ }^{\circ} \mathrm{C}$ with the Zetasizer Nano-ZS. The zetapotential of the samples was calculated from $\mu \mathrm{E}$ using following Smoluchowski's equation:

$$
\mu \mathrm{E}=\frac{\zeta \varepsilon}{\eta}
$$

where $\zeta$ is the zeta-potential, $\varepsilon$ the permittivity of solvent, and $\eta$ the solvent viscosity [23].

\section{Transmission electron microscopy}

$10 \mu \mathrm{L}$ of $1 \mathrm{mM}$ liposomes were dropped on 200 mesh copper grids (Electron Microscopy Sciences, Hatfield, PA, USA) and dried at room temperature for $5 \mathrm{~h}$. Images were collected using the Phillips CM-10 transmission electron microscope at $80 \mathrm{kV}$, equipped with a 15 megabyte SIA digital camera.

\section{UV-Vis spectroscopy}

The absorption spectrum for the CF-750 encapsulated liposomes was analyzed via UV-Visible spectroscopy. Absorption at wavelengths every $10 \mathrm{~nm}$ between 650 and $860 \mathrm{~nm}$ was measured using the NanoDrop 2000 Spectrophotometer (Thermo Scientific, Waltham, MA, USA) and accompanying software. Near-infrared (NIR) fluorescence signal was confirmed using Li-Cor Odyssey infrared scanner (Li-Cor, Lincoln, NE, USA).

\section{Western blot analysis}

Whole cell lysates from S2VP10L, MiaPaCa-2, S2013, and SCC-1 cells were collected to determine IGF1R expression. The cells were plated at a density of $5 \times 10^{5}$ cells per well in a 6 -well plate $24 \mathrm{~h}$ prior to protein harvest. Cells were then lysed in a solution containing $1 \%$ NP-40, $1 \%$ protease inhibitor, and $1 \%$ phosphatase inhibitor (Thermo) in deionized water. The lysates were centrifuged at $13,000 \times g$ for $10 \mathrm{~min}$. Total protein concentration was determined via Bradford assay (Bio-Rad, Hercules, CA, USA).

Approximately $50 \mu \mathrm{g}$ of total protein was dissolved in deionized water, loading buffer, and reducing agent (Life Technologies, Carlsbad, CA, USA). Proteins were separated using NuPage 4-12 \% Bis-Tris gel and transferred onto a nitrocellulose membrane by iBlot (Life Technologies). The membrane was blocked in blocking buffer (Li-Cor) for $30 \mathrm{~min}$ and then incubated overnight at $4{ }^{\circ} \mathrm{C}$ with IGF1R antibody (Abcam, Cambridge, England) at a concentration of $1 \mu \mathrm{g} / \mathrm{mL}$ and $\beta$-Actin antibody (Thermo) at a concentration of 1:3000. The membrane was then washed $3 \times$ with TBS $(20 \mathrm{mM}$ Tris$\mathrm{HCl}, 150 \mathrm{mM} \mathrm{NaCl}$ in $\mathrm{diH}_{2} \mathrm{O}$ ) for $10 \mathrm{~min}$ each, incubated with donkey anti-mouse IRDye 680RD and donkey antirabbit IRDye $800 \mathrm{CW}$ secondary antibodies (Li-Cor) for $1 \mathrm{~h}$, washed $3 \times$ with TBS for $10 \mathrm{~min}$ each, and scanned using Li-Cor Odyssey infrared scanner. Dosimetry was performed using Li-Cor software.

\section{Immunocytochemistry}

S2VP10L and SCC-1 cells were each seeded into 3 wells of a chamber slide at a density of $3 \times 10^{5}$ cells/well. Cells were grown overnight in RPMI with $10 \% \mathrm{FBS}$ and $1 \%$ L-Glutamine at $37{ }^{\circ} \mathrm{C}$ with $5 \% \mathrm{CO}_{2}$. The cells were then serum-starved for $3 \mathrm{~h}$ by switching to RPMI with $1 \%$ BSA (Fisher). Immunocytochemistry buffers were prepared as follows: $\mathrm{PBS}^{++}\left(0.5 \mathrm{mM} \mathrm{CaCl}\right.$ and $\mathrm{MgCl}_{2}$ in PBS), $\mathrm{PBS}^{++++}$(97.9 mL PBS++, $2 \mathrm{~mL} 10 \% \mathrm{BSA}+$ $90.08 \mathrm{mg}$ dextrose), and citrate buffer (1 mL $20 \times$ SCC

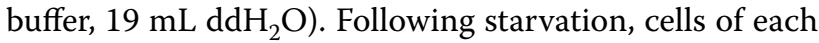
line were treated with $30 \mu \mathrm{L}$ PBS (control), $30 \mu \mathrm{L}$ of $1 \mathrm{mM}$ naked liposomes, or $30 \mu \mathrm{L}$ of $1 \mathrm{mM} \mathrm{Sdc1}$ liposomes for $3 \mathrm{~h}$. Then, the wells were washed $2 \times$ with cold $\mathrm{PBS}^{++++}$, $3 \times$ with ice cold citrate buffer, and again $2 \times$ with cold $\mathrm{PBS}^{++++}$. Cells were fixed with $4 \%$ paraformaldehyde (Electron Microscopy Sciences) for 5 min at room temperature and $15 \mathrm{~min}$ at $4{ }^{\circ} \mathrm{C}$. Next, the cells were washed $6 \times$ with $\mathrm{PBS}^{++}$for $10 \mathrm{~min}$ each. The slides were mounted in ProLong with DAPI (Invitrogen, Waltham, MA, USA) and set overnight. Images were taken at $400 \times$ magnification with a Zeiss Image Photomicroscope 2 (Carl Zeiss, Oberkochen, Germany) using DAPI, FITC, and Texas Red filters. The exposure times used were $20 \mathrm{~ms}$ DAPI, $200 \mathrm{~ms}$ FITC, and $200 \mathrm{~ms}$ Texas Red.

\section{Orthotopic pancreatic cancer xenografts}

Strict adherence to the University of Louisville Institutional Animal Care and Use Committee (IACUC)approved protocol was maintained throughout the study. Five-week old female CB-17 SCID mice (Harlan Laboratories, Indianapolis, IN, USA) were acclimated for 1 week prior to the study. Mice were anesthetized for all surgical procedures with $1.6 \%$ isoflurane and all procedures were performed in a sterile hood. Orthotopic cell implantation was performed as previously described $[15,21]$. A 1-cm incision was made in the left upper abdominal quadrant and the spleen was located and used to indirectly 
position the tail of the pancreas, avoiding direct manipulation of the pancreas. The suspension of S2VP10L (Luc positive) cells in PBS was stored on ice and drawn up with a 28 -gauge needle. Five mice were each injected with $1.5 \times 10^{5} \mathrm{~S} 2 \mathrm{VP} 10 \mathrm{~L}$ (Luc positive) cells $(30 \mu \mathrm{L})$ into the tail of the pancreas. Sterile cotton-tipped applicators were used to cover the injection site for $30 \mathrm{~s}$ to prevent peritoneal leakage. The organs were repositioned in the abdomen and the incision was closed using a single-layer closure with 5-0 nylon sutures. The mice recovered on a heated pad while receiving clear liquid acetaminophen for $24 \mathrm{~h}$ with food and water ad libitum. Confirmation of orthotopic implantation was performed using bioluminescence optical imaging on the Advanced Molecular Imager AMI-1000X (Spectral Imaging Instruments, Tucson, AZ, USA). Mice with detectable leakage from the pancreas were removed from the study. Mice received intraperitoneal (IP) injection of $2.5 \mathrm{mg}$ luciferin $10 \mathrm{~min}$ prior to weekly imaging to monitor orthotopic tumor and metastatic growth. Region of interest (ROI) analysis was used to measure light emitted for orthotopic sites using the AMI Image Viewer software. Sutures were removed 7 days following implantation. At 10 days post-implantation, mice were IV injected with $200 \mu \mathrm{L}$ of $100 \mathrm{nM}$ untargeted CF-750 or Sdc1-tagged CF-750 liposomes.

\section{In vivo imaging and reconstruction}

Multispectral optoacoustic tomographic (MSOT) imaging was performed as described by Kimbrough et al. [20]. Mice were anesthetized with $1.6 \%$ isoflurane and prepped for imaging with a combination of Nair cream with aloe (Church and Dwight Co., Princeton, NJ, USA) and shaving. The mice were imaged using MSOT system InVision TF 256 (iThera Medical, Munich, Germany). Serial slice images were taken in $0.2 \mathrm{~mm}$ steps between the diaphragm to the bottom of the kidneys $(40-55 \mathrm{~mm})$ at wavelengths of 680,710 , 730, 740, 760, 770, 780, 800, 850, and $900 \mathrm{~nm}$ using 25 averages per wavelength with acquisition time of $10 \mu \mathrm{s}$ per frame $[15,16]$ in order to minimize the influence of animal movement. Images were taken at $4,8,16$, and $24 \mathrm{~h}$ post injection to track liposome uptake and accumulation. Excitation of CF-750 liposomes was conducted using a tunable parametric oscillator pumped by an Nd:YAG laser. The pancreas tumor was identified by a live-feed screen preview multispectral signal. Videorate acquisition produced an imaging clip compiled from single-slice acquisition in less than $1 \mathrm{~ms}$, resulting in an image data rate of 10 frames per second. Image reconstruction was conducted using backprojection at a resolution of $75 \mu \mathrm{m}$. Multispectral processing was conducted using linear regression (ViewMSOT 3.5). A $3.5 \mathrm{~mm}$ diameter ellipse was used for region-of-interest analysis of liposome signal for tumor, liver, and kidney as in [15].

\section{Ex vivo imaging of organs}

Mice were euthanized $24 \mathrm{~h}$ after liposomal injections via carbon dioxide inhalation. The pancreas, liver, and spleen were harvested and imaged ex vivo to compare accumulation of naked and Sdc1 tagged liposomes from each of the five mice imaged using MSOT. The organs were imaged using near-infrared (NIR) fluorescence with the AMI-1000X at $675 \mathrm{~nm}$ excitation and $760 \mathrm{~nm}$ emission.

\section{Results and discussion \\ Western blot}

Western blot analysis was performed to evaluate the expression of IGF1-R on the three different pancreatic adenocarcinoma cell lines (S2VP10L, MiaPaCa-2, and S2013). The head and neck squamous cell carcinoma cell line SCC-1 served as a negative control. IGF1R plays an important role in cell proliferation, tumor metastasis, and anti-apoptotic pathways and thus is highly expressed in aggressive cancers. High IGF1R expression correlates to resistance to chemotherapy and radiation-induced apoptosis and is a marker for poor prognosis in pancreatic, breast, lung, and prostate cancers [24-29].

As expected, SCC-1 cells expressed much lower levels of IGF1R than pancreatic cancer lines (Fig. 1a). Less aggressive MiaPaCa- 2 cells also had relatively low IGF1R expression. Highly aggressive and metastatic S2VP10L and S2013 cells had five- and seven-fold increase of IGF1R expression, respectively compared to SCC-1 cells (Fig. 1b). S2VP10L cells were chosen as the pancreatic cell line to be used for the remainder of the experiments due to their predictable behavior in vivo and relatively high levels of IGF1R expression.

Syndecan-1 (Sdc1) is a transmembrane heparin sulfate proteoglycan that plays a role in cell proliferation and migration. The ectodomain of Sdc1 interacts with IGF1R to activate $\alpha_{5} \beta_{3}$ integrin, a mechanism not found in non-malignant epithelial cells [24, 30, 31]. High IGF1R expression in aggressive cancers coupled with the Sdc1IGF1R $-\alpha_{5} \beta_{3}$ integrin interaction makes targeting IGF1R via Sdc1 an attractive option for a theranostic nanoparticle for pancreatic adenocarcinoma.

\section{Characterization of liposomes}

Liposomes were synthesized to encapsulate CF-750 dye or propidium iodide and tagged with Syndecan-1. Transmission electron microscopy (TEM) of the liposomes shows the average size to be $117 \mathrm{~nm}$ (Fig. 2c). The size of liposomes observed by TEM is smaller than DLS (129 nm, Fig. 2a), as TEM yields a number-averaged size whereas DLS does a Z-averaged size that takes into 


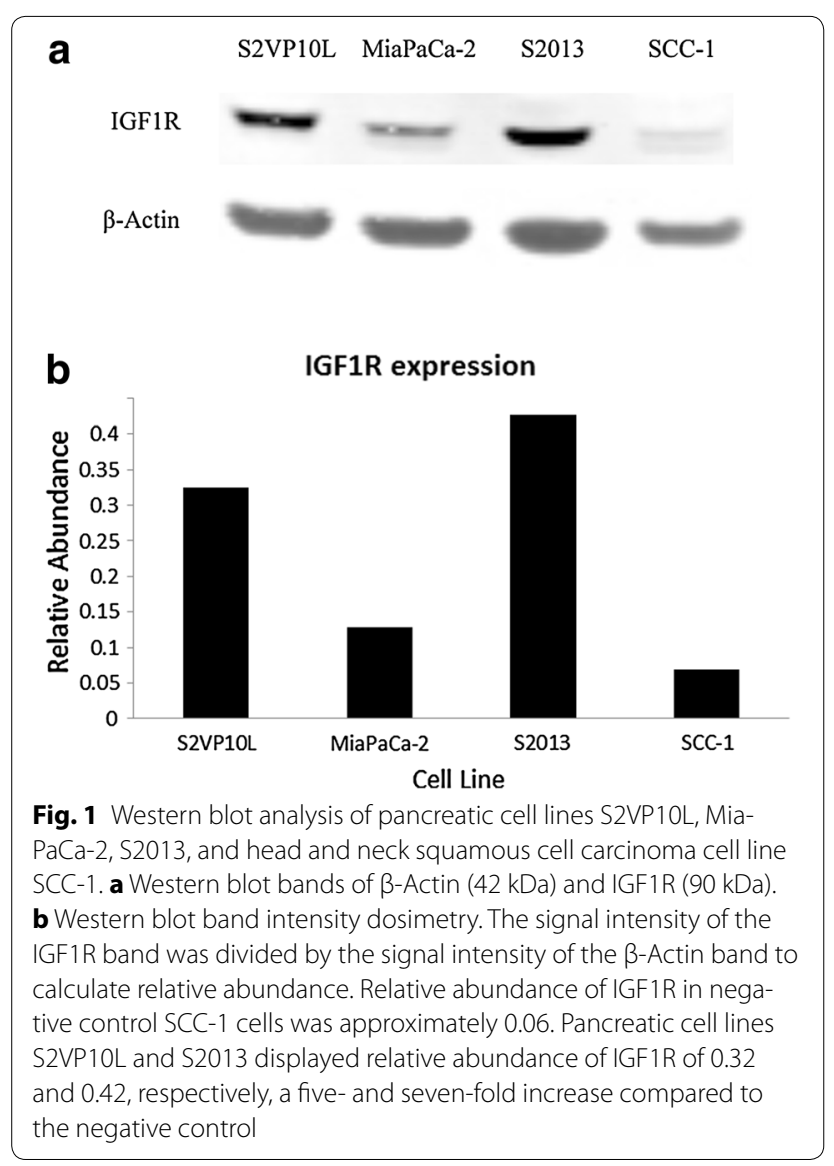

account hydration layers. Based on the low polydispersion index, the liposomes are uniform in size (Fig. 2a). The liposomes also have a slight positive charge of $0.3 \mathrm{mV}$ (Fig. 2b).

The leaky vasculature and inadequate lymphatics around solid tumors encourages accumulation of nanoparticles of a particular size $(\sim 100 \mathrm{~nm})$, a phenomenon termed the enhanced permeability and retention effect (EPR) [32]. It is estimated that nanoparticles $60-150 \mathrm{~nm}$ are small enough to extravasate from the blood into the tumor interstitial space through these pores within the vasculature [33]. Slightly larger particles such as albuminbound chemotherapies with diameters around $130 \mathrm{~nm}$ also display EPR and are suitable for IV injection [34]. Liposomal nanocarriers such as Sdc1-tagged liposomes can use passive targeting via EPR to deliver therapeutic agents specifically to tumor cells while bypassing normal, healthy tissue. Treatment with liposome-encapsulated therapeutics have been shown bioavailability of therapeutic agents at the tumor site, reduces off-target toxicity, and increases circulation time [35, 36]. Additionally, liposomes are permeable in environments with high collagen content, a characteristic of the extracellular matrix of pancreatic tumors, making them particularly attractive nanoparticles for pancreatic adenocarcinoma [37, 38].

In this study, Syndecan-1 was conjugated to the liposomes to act as a targeting ligand, a molecule that

\section{a}

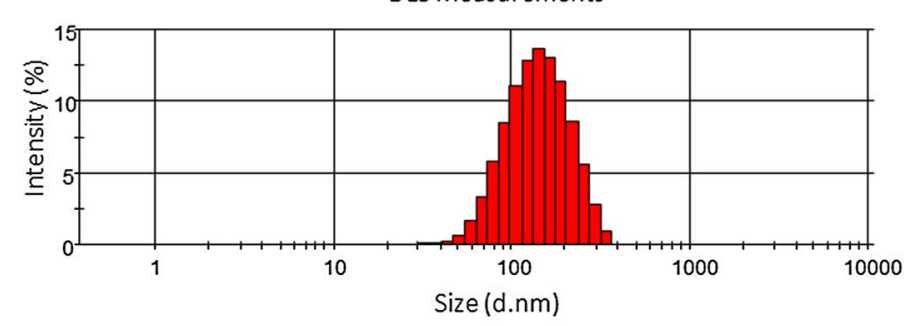

b

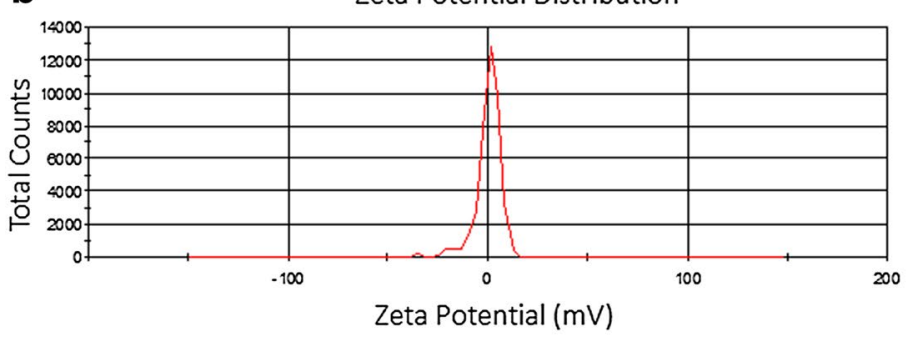

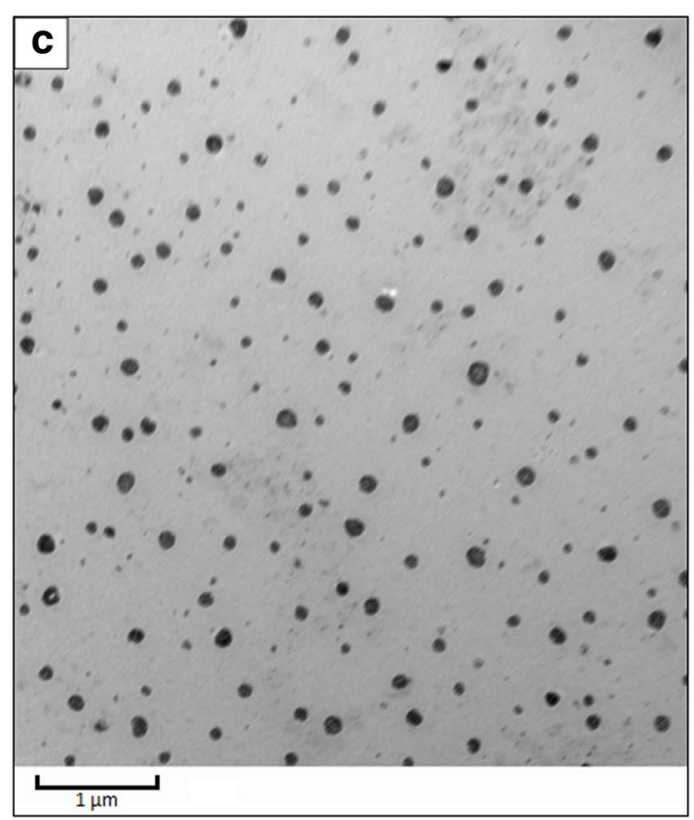

Fig. 2 a DLS measurements to determine the size of the liposomes. The liposomes are approximately 129 nm in diameter with a polydispersion index of 0.05. b Zeta-potential measurements of the liposomes. Z = $0.3 \mathrm{mV}$. c Transmission electron microscopy of the liposomes. The average size of the liposomes as determined by TEM is approximately $117 \mathrm{~nm}$ 
interacts with extracellular or trans-membrane molecules on cancer cells [19]. This allows Sdc1-tagged liposomes to take advantage of EPR and active targeting of IGF1R to enhance specificity for the tumor. Receptor-ligand targeting has been shown to improve target cell recognition within the tumor, leading to increased accumulation within cancer cells and increased cellular uptake when compared to non-targeted particles [39].

\section{UV-Vis spectroscopy}

The absorption spectrum for CF-750 encapsulated Sdc1 liposomes was analyzed using UV-Vis spectroscopy. The peak absorption of the liposomes was $750 \mathrm{~nm}$, the same as the original dye (Additional file 2: Figure S2). Thus, encapsulating the dye within liposomes does not change the optical behavior of the dye.

\section{Immunocytochemistry}

Immunocytochemistry slides of S2VP10L and SCC-1 cells were treated with non-targeted and Sdc1 liposomes containing propidium iodide and imaged using Texas Red, DAPI, and FITC filters. Fluorescence seen using the
Texas Red filter indicates propidium iodide (PI) uptake by the cell. In IGF1R-positive S2VP10L cells, we found greatly increased uptake of PI when treated with Sdc1tagged liposomes (Fig. 3c) compared to naked liposomes (Fig. 3b). Treatment with non-targeted liposomes does not result in the intracellular localization of dye, as the green fluorescence indicates extracellular accumulation (Fig. 3e) rather than intracellular uptake. The bright yellow areas in Fig. 4f indicate locations where red and green light are both present, suggesting PI on both the cell surface as well as within the cytoplasm, where it is likely bound to mitochondrial DNA or free-floating RNA. White areas indicate red, green, and blue co-localization demonstrating the PI binding to nuclear DNA. Untreated S2VP10L cells show minimal red autofluorescence (Fig. 3a) and no green autofluorescence (Fig. 3d).

Propidium iodide, when bound to nucleic acids, displays maximum excitation and emission values at 538 and $617 \mathrm{~nm}$, respectively and fluoresces red using the Texas Red filter. However, PI in its free form exhibits different optical characteristics: maximum excitation at $488 \mathrm{~nm}$ and emission at $590 \mathrm{~nm}$ [40], fluorescing green
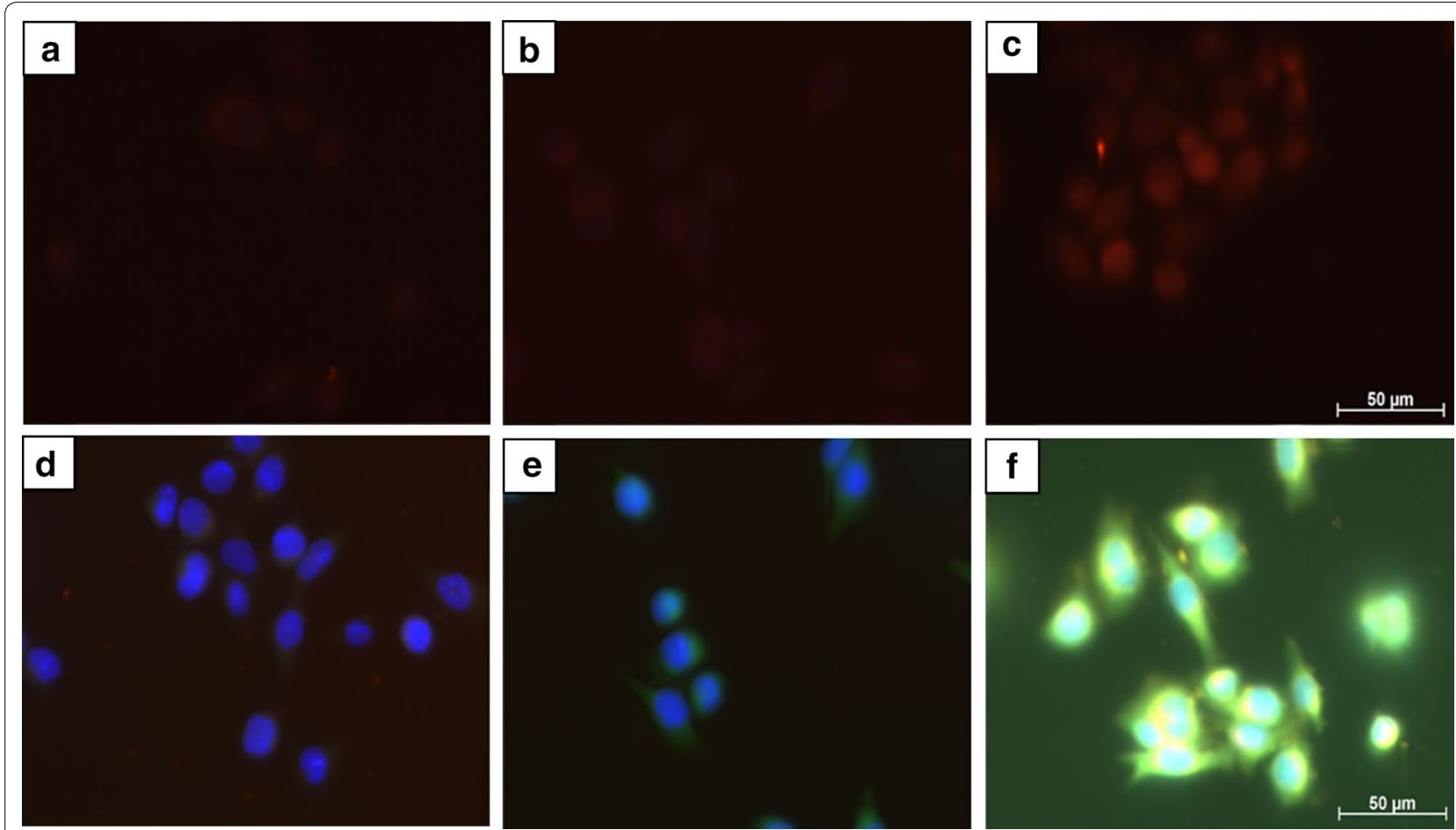

Fig. 3 S2VP10L cells following $3 \mathrm{~h}$ treatment at $\times 400$ magnification. All images were taken with the same exposure times. Top row a-c was taken with the Texas Red filter. Bottom row d-f was taken with Texas Red, DAPI, and FITC filters. a Untreated control cells show minimal red autofluorescence. $\mathbf{b}$ Cells were treated with non-targeted liposomes display faint red signal, corresponding to minimal dye uptake. c Cells were treated with Sdc1 liposomes and a much stronger red fluorescence, indicating uptake of PI and binding of PI to DNA is observed. d Control cells with DAPI. e Cells treated with non-targeted liposomes. Green signal is due to unbound PI, showing dye accumulation outside of the cell. $\mathbf{f}$ Cells treated with Sdc1 liposomes. Yellow signal occurs due to co-localization of red and green fluorescence, indicating that dye was located both on the cell surface and in the cytoplasm. White signal is colocalization of red, green, and DAPI signal 
using the FITC filter. This allows for distinction between excess dye bound on the cell surface versus internalized dye bound to nuclear or cytoplasmic nucleic acids.

S2VP10L cells treated with Sdc1 liposomes show dramatically increased PI uptake compared to cells treated with non-targeted liposomes. The Sdc1-IGF1R interaction may facilitate the liposomes to release their contents into the cell. Since this mechanism is specific to IGF1Rpositive cells, chemotherapeutic agents may be loaded into Sdc1 liposomes and delivered only to tumor cells. Using a nanocarrier of this sort can reduce the incidence and intensity of side effects, which results in improved patient compliance and prognosis $[6-8,10]$. Furthermore, the presence of a contrast agent allows for tracking of liposomal movement and accumulation and can be used to diagnose pancreatic cancer. Thus, Sdc1-tagged liposomes have the potential to fulfill both diagnostic and therapeutic functions.

\section{In vivo imaging and reconstruction}

Syndecan-1-tagged liposomes were injected 10 days post-implantation of the tumor. Liposome accumulation in vivo was determined using MSOT imaging at 4, 8, 16, $24 \mathrm{~h}$ post injection. Representative location of organs as seen on the MSOT can be viewed in Supplemental Fig. 3. Liposome accumulation in the tumor peaked at $8 \mathrm{~h}$ post-injection and declined within $24 \mathrm{~h}$ (Fig. 4c). The highest region of photoacoustic signal was between 45 and $46 \mathrm{~mm}$ in the mouse, and corresponded with the location of the pancreatic tumors (Fig. 4a). Low signal
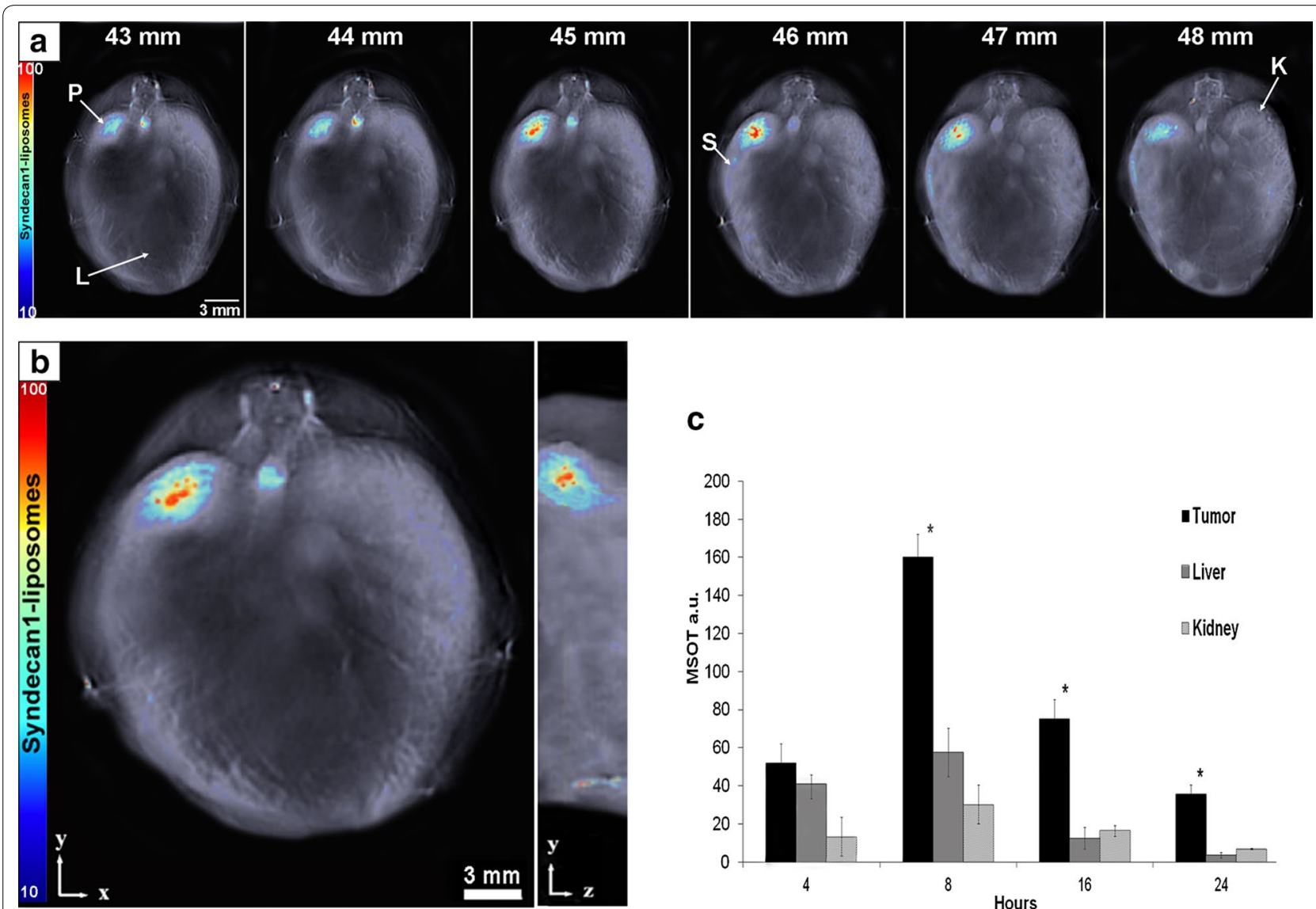

C
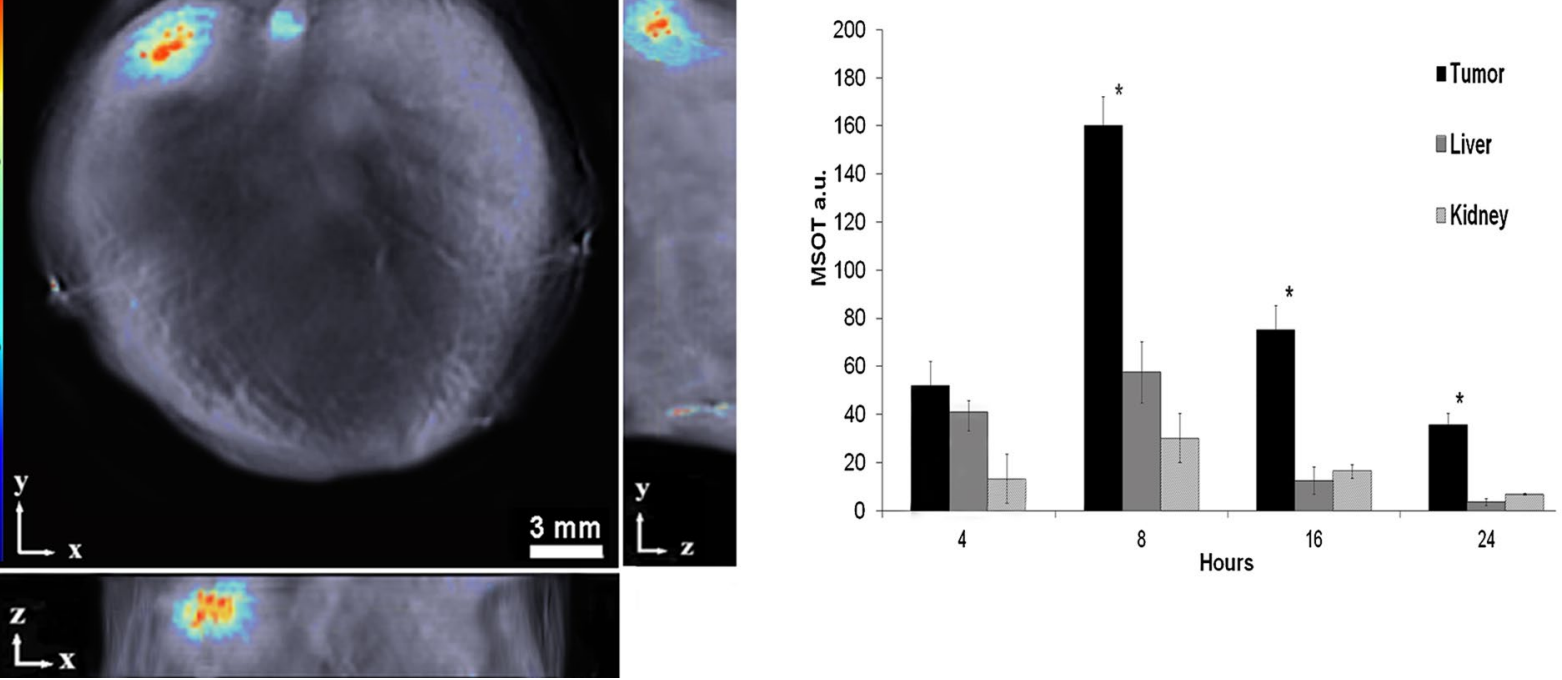

Fig. 4 a Serial slice images of liposomal accumulation taken from 43 to $48 \mathrm{~mm}$ (abdomen). The highest signal intensity of the liposomes was at 45-46 mm. Organs are identified: P pancreas tumor, L liver, $S$ spleen, K kidney. b Orthogonal views of the pancreatic tumor and liposomal accumulation through different anatomical planes. c ROI analysis on liposome signal in various locations over time measured in MSOT a.u. Bar height represents the median value and error bars represent the standard deviation throughout the organ. Peak liposomal accumulation occurred at $8 \mathrm{~h}$ post-injection. Significantly more liposomes accumulated in the tumor versus off-target organs ( $p<0.05)$. Representative locations of organs can be viewed in Additional file 3: Figure S3 
intensity was observed in the spleen and liver (Fig. 4b), indicating significantly more liposomes accumulated in the tumor versus in the liver and kidney $(\mathrm{p}<0.05)$. Furthermore, orthogonal views of the tumor demonstrate probe accumulation along three spatial dimensions, and suggest liposome penetration and accumulation within the interior of the pancreatic tumor (Fig. 4b). Error bars represent standard deviation throughout the organ (Fig. 4c).

The ability to utilize targeting ligands or monoclonal antibodies to improve tumor specific uptake of nanoparticles for both imaging and treatment of cancer is one of the most important aspects to successfully translate nanotechnology to the clinic. This is especially important as non-targeted liposomal formulations of chemotherapeutics, i.e. doxil, have failed to demonstrate significantly increased tumor localization in the clinic [41]. The use of Syndecan-1 ligand to target the liposomes to the pancreatic tumor along with low off-target binding demonstrates the feasibility of this approach. The ability of these particles to contain contrast agents can allow for alternative methods of imaging pancreatic cancer using current imaging technology. Previous studies have shown that liposome-encapsulated contrast agents enhance imaging of tumors and inflammatory lesions using CT and SPECT by increasing circulation time [42, 43]. Modifying the lipid composition of Sdc1-tagged liposomes in this way could enhance the use of MRI for pancreatic adenocarcinoma by allowing for the imaging of smaller tumors. Alternatively, Sdc1-tagged or similar liposomes specific to pancreatic cancer can be loaded with a contrast dye to better image suspected pancreatic lesions using CT, improving an already-established method of imaging pancreatic cancer.

Photoacoustic imaging is capable of producing high resolution molecular images in vivo based on the absorption of contrast agents. MSOT technology, while new, is being established in clinical settings with the development of a handheld MSOT system [44]. In humans, MSOT has been able to provide high-resolution and high-contrast imaging of both endogenous (such as oxy- and deoxy-hemoglobin, and melanin in the monitoring of melanoma) and exogenous (NIR dyes) chromophores in the context of cancer [45-47]. Currently, photoacoustic imaging has been demonstrated at depths of up to $5 \mathrm{~cm}$ while volumetric real-time photoacoustic imaging image has been demonstrated depths of

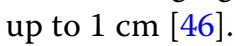

The use of photoacoustic imaging of pancreatic cancer is currently limited by inability to deliver the laser pulse to deep organs. However, photoacoustic endoscopy, currently in the preclinical stage, is emerging as a new modality for imaging the GI tract [48]. Endoscopic ultrasound is currently used for diagnosis of pancreatic cancer via ultrasound-guided needle biopsies of suspected tumors [4]. With further technological advancement, photoacoustic endoscopy can deliver light through the lining of the stomach to the pancreas. If used to image pancreatic adenocarcinoma-specific chromophores, such as dyes encapsulated within targeted liposomes, photoacoustic endoscopy has the potential to become a non-invasive method of diagnosing pancreatic cancer.

\section{Ex vivo imaging of organs}

Ex vivo fluorescent imaging confirms accumulation of Sdc1-liposomes within organs (Fig. 5). Mice were euthanized $24 \mathrm{~h}$ post-liposomal injection. The pancreas, liver, and spleen were imaged using NIR fluorescent imaging with excitation $675 \mathrm{~nm}$ and emission $760 \mathrm{~nm}$. Sdc1-tagged liposomes bound preferentially to the pancreas tumor with little off-target binding in the liver and spleen. While some naked liposomes reached the pancreatic tumor, this was likely due to passive targeting from EPR and the leakiness of the blood vessels around the tumor [32]. Non-targeted liposomes accumulated primarily within the liver.

The dramatic difference in liposome localization and specificity was due to the presence of the targeting ligand, Syndecan-1. Sdc1 provided a mechanism for the liposomes to actively target pancreatic cancer by binding to IGF1R, overexpressed on S2VP10L cells (Fig. 1). Active targeting enhanced the specificity of the liposomes for pancreatic cancer when compared to untargeted liposomes, which is in congruence with previous studies [39].

\section{Conclusion}

Syndecan-1 tagged liposomes actively target pancreatic adenocarcinoma with minimal off-target binding in vivo. Using liposomes that contained contrast agents allowed for non-invasive tracking via photoacoustic imaging, and MSOT was able to provide high-resolution molecular images of our Syndecan-1 liposomes in an orthotopic pancreatic xenograft mouse model. Ex vivo analysis of non-targeted and Syndecan-1 liposomes show that unlike naked liposomes, Syndecan-1 liposomes do not primarily accumulate in off-target organs such as the liver. In this study, the liposomes only contained a fluorescent dye for diagnostic imaging; in the future, these liposomes may serve as a nanocarrier for chemotherapeutic agents. Our in vitro results demonstrate improved intracellular delivery of liposomal content into tumor cells using targeted liposomes. Future studies should explore the ability of Syndecan-1 liposomes to preferentially release drugs at the tumor site and compare their effectiveness to current 


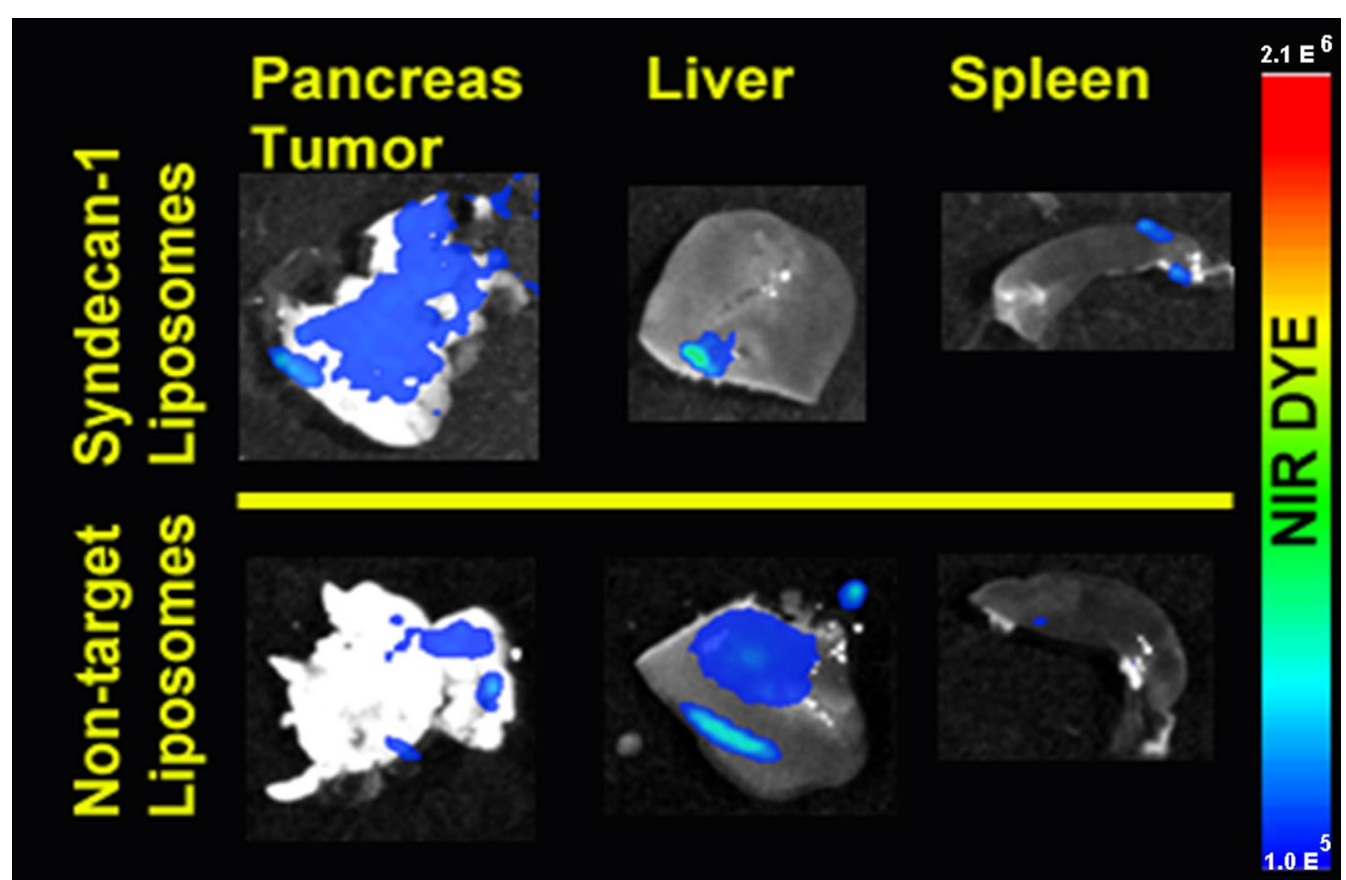

Fig. 5 Ex vivo fluorescent imaging of the pancreas, liver, and spleen $24 \mathrm{~h}$ post-injection. Sdc1 liposomes accumulated in the tumor with very little off-target binding in the liver and spleen. Non-targeted liposomes tend to accumulate in the liver with low signal in the tumor

treatments for pancreatic cancer. Ultimately these studies may lead to the development of a theranostic nanoparticle for clinical use.

\section{Additional files}

Additional file 1: Figure S1. Structures of the lipids used for control and Sdc1-tagged liposome synthesis.

Additional file 2: Figure S2. Absorption spectrum for CF-750 encapsulated Sdc1 liposomes. The liposomes demonstrated fluorescence activity with peak absorbance at $750 \mathrm{~nm}$. Encapsulating the CF-750 dye within the Sdc1 liposomes did not change the optical activity of the dye.

Additional file 3: Figure S3. Representative locations of organs on MSOT at both 46 and $49 \mathrm{~mm}$. Organs are noted PT = Pancreas tumor, $\mathrm{S}=$ Spleen, $\mathrm{L}=$ Liver, $\mathrm{BV}=$ Blood vessel, $\mathrm{K}=$ Kidney.

\section{Authors' contributions}

WY carried out evaluation of IGF1-R expression by western blot, construction and characterization of liposomes, assisted in the immunocytochemistry, and aided in the drafting of the manuscript. CWK and LRM carried out the in vivo experiments including orthotopic mouse injection, IV liposome injection in vivo imaging, and drafting of the manuscript. JG G-G and CTB participated in characterization of the liposome and drafting the manuscript. PC aided in the characterization and construction of the liposomes. WEG participated in the immunocytochemistry experiments and acquisition of images. LRM conceived of the study and coordinated the experiments. All authors read and approved the final manuscript.

\section{Author details}

${ }^{1}$ University of Louisville, 505 S Hancock, Louisville, KY 40202, USA. ${ }^{2}$ University of Alabama Birmingham, ZRB 408, 1720 2nd Avenue South, Birmingham, AL 35294, USA.

\section{Acknowledgements}

This work was funded in part by the Rounsavall Foundation the University of Louisville School of Medicine and the UAB/UMN SPORE in Pancreatic Cancer: Tissue Resources and Molecular Pathology Core 2P50CA101955.

\section{Competing interests}

The authors declare that they have no competing interests.

Received: 29 July 2015 Accepted: 19 October 2015

Published online: 01 December 2015

\section{References}

1. Li D, Xie K, Wolff R, Abbruzzese JL. Pancreatic cancer. Lancet. 2004:363(9414):1049-57.

2. Howlader N, Noone AM, Krapcho M, Garshell J, Neyman N, Altekruse SF, Kosary CL, Yu M, Ruhl J, Tatalovich Z, Cho H, Mariotto A, Lewis DR, Chen HS, Feuer EJ, Cronin KA (eds) SEER cancer statistics review, 1975-2010, National Cancer Institute. Bethesda, MD. http://seer.cancer.gov/ csr/1975_2010/, based on November 2012 SEER data submission, posted to the SEER web site, April 2013.

3. Burris HA 3rd, Moore MJ, Andersen J, Green MR, Rothenberg ML Modiano MR, Cripps MC, Portenoy RK, Storniolo AM, Tarassoff P, Nelson $R$, Dorr FA, Stephens CD, Von Hoff DD. Improvements in survival and clinical benefit with gemcitabine as first-line therapy for patients with advanced pancreas cancer: a randomized trial. J Clin Oncol. 1997:15(6):2403-13.

4. Agarwal B, Abu-Hamda E, Molke KL, Correa AM, Ho L. Endoscopic ultrasound-guided fine needle aspiration and multidetector spiral $\mathrm{CT}$ in the diagnosis of pancreatic cancer. Am J Gastroenterol. 2004;99(5):844-50.

5. Long J, Zhang Y, Yu X, Yang J, LeBrun DG, Chen C, Yao Q, Li M. Overcoming drug resistance in pancreatic cancer. Expert Opin Ther Targets. 2011;15(7):817-28. 
6. Patra CR, Bhattacharya R, Wang E, Katarya A, Lau JS, Dutta S, Muders M, Wang S, Buhrow SA, Safgren SL, Yaszemski MJ, Reid JM, Ames MM, Mukherjeel P, Mukhopadhyay D. Targeted delivery of gemcitabine to pancreatic adenocarcinoma using cetuximab as a targeting agent. Cancer Res. 2008;68(6):1970-8.

7. He Y, Zhang L, Zhu D, Song C. Design of multifunctional magnetic iron oxide nanoparticles/mitoxantrone-loaded liposomes for both magnetic resonance imaging and targeted cancer therapy. Int J Nanomedicine. 2014;22(9):4055-66

8. Schleich N, Po C, Jacobs D, Ucakar B, Gallez B, Danhier F, Préat V. Comparison of active, passive and magnetic targeting to tumors of multifunctional paclitaxel/SPIO-loaded nanoparticles for tumor imaging and therapy. J Control Release. 2014;28(194):82-91.

9. Lee RJ, Low PS. Delivery of liposomes into cultured KB cells via folate receptor-mediated endocytosis. J Biol Chem. 1994;269(5):3198-204.

10. Rizzo LY, Theek B, Storm G, Kiessling F, Lammers T. Recent progress in nanomedicine: therapeutic, diagnostic, and theranostic applications. Curr Opin Biotechnol. 2013;24(6):1159-66.

11. Fang C, Zhang M. Nanoparticle-based theragnosticss: integrating diagnostic and therapeutic potentials in nanomedicine. J Control Release. 2010;146(1):2-5.

12. Ryu JH, Koo H, Sun IC, Yuk SH, Choi K, Kim K, Kwon IC, Fang C, Miqin Z. Tumor-targeting multi-functional nanoparticles for theragnosis: new paradigm for cancer therapy. Adv Drug Deliv Rev. 2012;64(13):1447-58.

13. Cox B, Laufer JG, Arridge SR, Beard PC. Quantitative spectroscopic photoacoustic imaging: a review. Biomed Opt. 2012;17(6):061202. doi:10.1117/1.JBO.17.6.061202.

14. Razansky D, Deliolanis NC, Vinegoni C, Ntziachristos V. Deep tissue optical and optoacoustic molecular imaging technologies for pre-clinical research and drug discovery. Curr Pharm Biotechnol. 2012;13(4):504-22.

15. Kimbrough CW, Khanal A, Zeiderman M, Khanal BR, Burton NC, McMasters KM, Vickers SM, Grizzle WE, McNally LR. Targeting acidity in pancreatic adenocarcinoma: multispectral optoacoustic tomography detects ph-low insertion peptide probes in vivo. Clin Cancer Res. 2015;21(20):4576-85

16. Buehler A, Herzog E, Ale A, Smith BD, Ntziachristos V, Razansky D. High resolution tumor targeting in living mice by means of multispectral optoacoustic tomography. EJNMMI Res. 2012;2:14.

17. Lutzweiler C, Razansky D. Optoacoustic imaging and tomography: reconstruction approaches and outstanding challenges in image performance and quantification. Sensors. 2013;13(6):7345-84.

18. Ntziachristos $\vee$, Razansky D. Molecular imaging by means of multispectral optoacoustic tomography (MSOT). Chem Rev. 2010;110(5):2783-94.

19. Lammers T, Kiessling F, Hennink WE, Storm G. Drug targeting to tumors: principles, pitfalls and (pre-) clinical progress. J Control Release. 2012;161(2):175-87

20. Kimbrough CW, Hudson S, Khanal A, Egger ME, McNally LR. Orthotopic pancreatic tumors detected by optoacoustic tomography using Syndecan-1. J Surg Res. 2015;193(1):246-54.

21. McNally LR, Welch DR, Beck BH, Stafford LJ, Long JW, Sellers JC. KISS1 over-expression suppresses metastasis of pancreatic adenocarcinoma in a xenograft mouse model. Clin Exp Metastasis. 2010;27(8):591-600.

22. Dua JS, Rana AC, Bhandari AK. Liposome: methods of preparation and applications. Int J Pharm Sci Res. 2012;3(2):14-20.

23. Khanal A, Ullum C, Kimbrough CW, Garbett NC, Burlison JA, McNally MW, Chuong P, El-Baz AS, Jasinski JB, McNally LR. Tumor targeted mesoporous silica-coated gold nanorods facilitate detection of pancreatic tumors using Multispectral optoacoustic tomography. Nano Res. 2015. doi:10. 1007/s12274-015-0886-8.

24. Ouban A, Muracab P, Yeatman T, Coppola D. Expression and distribution of insulin-like growth factor-1 receptor in human carcinomas. Hum Pathol. 2003;34(8):803-8.

25. Turner BC, Haffty BG, Narayanan L, Yuan J, Havre PA, Gumbs AA, Kaplan L, Burgaud JL, Carter D, Baserga R, Glazer PM. Insulin-like growth factor-I receptor overexpression mediates cellular radioresistance and local breast cancer recurrence after lumpectomy and radiation. Cancer Res. 1997:57(15):3079-83.

26. Hirakawa T, Yashiro M, Murata A, Hirata K, Kimural K, Amano R, Yamadal N, Nakata B, Hirakawa K. IGF-1 receptor and IGF binding protein-3 might predict prognosis of patients with resectable pancreatic cancer. BMC Cancer. 2013;13:392
27. Valsecchi ME, McDonald M, Brody JR, Hyslop T, Freydin B, Yeo CJ, Solomides C, Peiper SC, Witkiewicz AK. Epidermal growth factor receptor and insulin like growth factor 1 receptor expression predict poor survival in pancreatic ductal adenocarcinoma. Cancer. 2012;118(14):3484-93.

28. Jones HE, Goddard L, Gee JM, Hiscox S, Rubini M, Barrow D, Knowlden JM, Williams S, Wakeling AE, Nicholson RI. Insulin-like growth factor-I receptor signalling and acquired resistance to gefitinib (ZD1839; Iressa) in human breast and prostate cancer cells. Endocr Relat Cancer. 2004;11(4):793-814.

29. Hellawell GO, Turner GDH, Davies DR, Poulsom R, Brewster SF, Macaulay VM. Expression of the type 1 insulin-like growth factor receptor is up-regulated in primary prostate cancer and commonly persists in metastatic disease. Cancer Res. 2002;62(10):2942-50.

30. Beauvais DM, Burbach BJ, Rapraeger AC. The syndecan-1 ectodomain regulates alphavbeta3 integrin activity in human mammary carcinoma cells. J Cell Biol. 2004;167:171-81.

31. Beauvais DM, Rapraeger AC. Syndecan-1 couples the insulin-like growth factor-1 receptor to inside-out integrin activation. J Cell Sci. 2010;123(21):3796-807.

32. Maeda H, Greish K, Fang J. The EPR effect and polymeric drugs: a paradigm shift for cancer chemotherapy in the 21 st century. Satchi-Fainaro R, Duncan R, editors. Polymer therapeutics II, vol 193. Berlin, Heidelberg: Springer; 2006. p. 103-21.

33. Prabhakar U, Maeda H, Jain RK, Sevick-Muraca EM, Zamboni W, Farokhzad OC, Barry ST, Gabizon A, Grodzinski P, Blakey DV. Challenges and key considerations of the enhanced permeability and retention effect for nanomedicine drug delivery in oncology. Cancer Res. 2013;73(8):2412-7.

34. Kim DH, Moon C, Oh SS, Park S, Jeong JW, Kim S, Lee HG, Kwon HJ, Kim KD. Liposome-encapsulated CpG enhances antitumor activity accompanying the changing of lymphocyte populations in tumor via intratumoral administration. Nucleic Acid Ther. 2015;25(2):95-102.

35. Andey TA, Sudhakar G, Marepally SK, Patel AR, Banerjee R, Sachdeva MS. Lipid nanocarriers of a lipid-conjugated estrogenic derivative inhibit tumor growth and enhance cisplatin activity against triple-negative breast cancer: pharmacokinetic and efficacy evaluation. Mol Pharm. 2015;12(4):1105-20

36. Kroon J, Buijs JT, van der Horst $G$, Cheung $H$, van der Mark $M$, van Bloois $L$, Rizzo LY, Lammers T, Pelger RC, Storm G, van der Pluijm G, Metselaar JM. Liposomal delivery of dexamethasone attenuates prostate cancer bone metastatic tumor growth in vivo. Prostate. 2015;75(8):815-24.

37. Yokoi K, Kojic M, Milosevic M, Tanei T, Ferrari M, Ziemys A. Capillary-wall collagen as a biophysical marker of nanotherapeutic permeability into the tumor microenvironment. Cancer Res. 2014;74(16):4239-46.

38. Hidenori M, Tatsuya M, Kunihiro T, Hiromu S, Hidenori M, Seiki T. Tumorstroma interaction of human pancreatic cancer: acquired resistance to anticancer drugs and proliferation regulation is dependent on extracellular matrix proteins. Pancreas. 2004;28(1):38-44.

39. Watanabe K, Kaneko M, Maitani Y. Functional coating of liposomes using a folate- polymer conjugate to target folate receptors. Int J Nanomedicine. 2012;7:3679-88.

40. Krishan A, Ganapathi RN, Israel M. Effect of adriamycin and analogs on the nuclear fluorescence of propidium iodide-stained cells. Cancer Res. 1978:38(11 Pt 1):3656-62.

41. Syrigos KN, Michalaki B, Alevyzaki F, Machairas A, Mandrekas D, Kindilidis K, Karatzas G. A phase-Il study of liposomal doxorubicin and docetaxel in patients with advanced pancreatic cancer. Anticancer Res. 2002;22(6B):3583-8.

42. Zheng J, Allen C, Serra S, Vines D, Charron M, Jaffray DA. Liposome contrast agent for CT-based detection and localization of neoplastic and inflammatory lesions in rabbits: validation with FDG-PET and histology. Contrast Media Mol Imaging. 2010;5(3):147-54

43. Chen MH, Chang CH, Chang YJ, Chen LC, Yu CY, Wu YH, Lee WC, Yeh CH, Lin FH, Lee TW, Yang CS, Ting G. MicroSPECT/CT imaging and pharmacokinetics of 188Re-(DXR)-liposome in human colorectal adenocarcinoma-bearing mice. Anticancer Res. 2010;30(1):65-72.

44. Buehler A, Kacprowicz M, Taruttis A, Ntziachristos V. Real-time handheld multispectral optoacoustic imaging. Opt Lett. 2013;38(9):1404-6.

45. Mallidi S, Luke GP, Emelianov S. Photoacoustic imaging in cancer detection, diagnosis, and treatment guidance. Trends Biotechnol. 2011;29(5):213-21. 
46. Deán-Ben XL, Razansky D. Functional optoacoustic human angiography with handheld video rate three dimensional scanner. Photoacoustics. 2013;1(3-4):68-73.

47. Heijblom M, Piras D, Xia W, van Hespen JC, Klaase JM, van den Engh FM, van Leeuwen TG, Steenbergen W, Manohar S. Visualizing breast cancer using the Twente photoacoustic mammoscope: what do we learn from twelve new patient measurements? Opt Express. 2012;20(11):11582-97.

48. Yoon TJ, Cho YS. Recent advances in photoacoustic endoscopy. World J Gastrointest Endosc. 2013;5(11):534-9.

Submit your next manuscript to BioMed Central and take full advantage of:

- Convenient online submission

- Thorough peer review

- No space constraints or color figure charges

- Immediate publication on acceptance

- Inclusion in PubMed, CAS, Scopus and Google Scholar

- Research which is freely available for redistribution

Submit your manuscript at

www.biomedcentral.com/submit

() BioMed Central 